\title{
The prognostic impact of neutrophil-to-lymphocyte ratio, lymphocyte-to-monocyte ratio, and platelet-to- lymphocyte ratio in patients with advanced colorectal cancer treated with first-line chemotherapy
}

\author{
Magdalena Krakowska ${ }^{1,2}$, Sylwia Dębska-Szmich ${ }^{1,2}$, Rafał Czyżykowski ${ }^{1,2}$, Anna Zadrożna-Nowak ${ }^{2}$, Piotr Potemski ${ }^{1,2}$ \\ ${ }^{1}$ Oncology Department, Chemotherapy Clinic, Medical University of Lodz, Lodz, Poland \\ ${ }^{2}$ Nicolaus Copernicus Multidisciplinary Centre for Oncology and Traumatology, Lodz, Poland
}

Gastroenterology Rev 2018; 13 (3): 218-222

DOI: https://doi.org/10.5114/pg.2018.78287

Key words: colorectal cancer, neutrophil-to-lymphocyte ratio, platelet-to-lymphocyte ratio, lymphocyte-to-monocyte ratio, complete blood count.

Address for correspondence: Magdalena Krakowska, Chemotherapy Clinic, Nicolaus Copernicus Multidisciplinary Centre for Oncology and Traumatology, 62 Pabianicka St, 93-513 Lodz, Poland, phone: +48 4268954 31, fax: +48 42689 54 32, e-mail: magdakrakowska@tlen.pl

\begin{abstract}
Introduction: Colorectal cancer is the second most frequently diagnosed malignancy and one of the leading causes of cancer-related death in Poland. Many reports of different types of cancer have indicated that blood count parameters may serve as a source of prognostic or predictive information.

Aim: To assess the association between these parameters and clinical outcome in patients with advanced colorectal cancer.

Material and methods: We retrospectively analysed a database of 295 patients with advanced colorectal cancer treated with first-line palliative chemotherapy at our institution from January 2008 to December 2012. Blood-based parameters were measured before the first cycle of treatment.

Results: The median progression-free survival (PFS) was 6.7 months, and the median overall survival was 17.6 months. A high neutrophil-to-lymphocyte ratio (NLR) and a high platelet-to-lymphocyte ratio (PLR) were associated with a shorter survival (hazard ratio (HR): 1.88, $p<0.0001$ for the NLR and HR: 1.39, $p=0.0054$ for the PLR), but for the PLR, we observed only a not significant trend toward a worse PFS ( $H R=1.25, p=0.07$ for the PLR and HR $=1.55, p=0.0004$ for the NLR). A high lymphocyte-to-monocyte ratio (LMR) was associated with a better prognosis $(H R=0.58, p \leq 0.0001)$ and a longer $P F S(H R=0.73$, $p=0.011$ ).

Conclusions: The blood-based parameters are readily available, reliable, and low-cost biomarkers, which can be easily incorporated into routine practice to predict the prognosis in patients with advanced colorectal cancer.
\end{abstract}

\section{Introduction}

Colorectal cancer (CRC) is the second most frequently diagnosed malignancy and one of the leading causes of cancer-related death in Poland. According to the National Cancer Registry in 2014, approximately 18,000 people developed CRC and 11,500 died because of it [1]. The median overall survival (OS) of those patients with unresectable metastatic CRC participating in recent clinical trials exceeds 30 months, and the prolongation of life depends mainly on a multidisciplinary strategic approach. The improvements in the efficacy of systemic treatment not only enable long-term disease control without a significant deterioration in the quality of life, but also result in facilitating the surgical resection of metastases or the use of ablative techniques [2]. However, despite considerable progress, important questions about the appropriate selection of patients for the multiple treatment options remain unanswered. To determine an optimal treatment strategy, it is important to evaluate the patient outcomes. Many prognostic and predictive factors are used in clinical practice, such as the performance status, number and localisation of the metastasis, and the serum carcinoembryonic antigen (CEA) level [2]. Recently, a growing number of reports have indicated that a routine blood count may serve 
as a source of important prognostic information. The parameters calculated on the basis of a complete blood count $(\mathrm{CBC})$, such as the neutrophil-to-lymphocyte ratio (NLR), lymphocyte-to-monocyte ratio (LMR), and platelet-to-lymphocyte ratio (PLR), could help to predict the clinical outcomes in different kinds of cancer without any additional costs [3-6].

\section{Aim}

The aim of this study was to evaluate the prognostic and predictive values of the CBC parameters in patients with advanced CRC treated with first-line chemotherapy.

\section{Material and methods Patient eligibility}

We retrospectively analysed a database of patients with CRC treated at our institution from January 2008 to December 2012. We selected patients who met the following criteria: available pathological diagnosis, evaluated as unresectable CRC, and treated with first-line palliative chemotherapy. The exclusion criterion was the lack of a CBC measured before the first cycle of systemic therapy.

This study was approved by the Ethics Committee of the Medical University of Lodz.

\section{Evaluations}

Detailed clinical data were collected, including each patient's sex, age, Eastern Cooperative Oncology Group (ECOG) performance status, primary tumour location, and presence and location of metastasis. We also analysed the data on the adjuvant and first-line palliative chemotherapy. Apart from a CBC, several other laboratory parameters were evaluated, such as the CEA and cancer antigen (CA) 19-9 levels, alkaline phosphatase $(\mathrm{ALP})$, and lactate dehydrogenase (LDH). All of the blood samples were taken before the first administration of chemotherapy. Based on the $C B C$, we calculated the NLR by dividing the absolute neutrophil count by the absolute lymphocyte count. Moreover, we calculated the $L M R$ and PLR in a similar way.

\section{Statistical analysis}

The PFS was measured from the date of the firstline palliative chemotherapy commencement to the date of disease progression or death, while the OS was measured from the date of the first-line palliative chemotherapy commencement to the date of death. The cut-off time for the survival follow-up data was December 2015. The PFS and OS of patients without disease progression or death were censored at the last contact date. The survival rates were calculated via the Kaplan-Meier method. Using the median as a cut-off, the patients were divided into those with low and those with high values of NLR, LMR, and PMR. Divisions of patients in respect of WBC, neutrophil, monocyte, lymphocyte, platelet counts, and haemoglobin concentration were based on normal values of the respective parameters. The relationship between the tested parameters and PFS or OS was analysed by a univariate Cox regression model. A $p$-value of $<0.05$ was considered to be significant in all of the analyses. All of the statistical analyses were performed with StatsDirect software for Windows (StatsDirect Ltd., Altrincham, UK).

\section{Results}

A total of 295 patients were included in this study. The median PFS was 6.7 months and the median OS was 17.6 months. During the follow-up period, 284 (96\%) patients died.

The patients were predominantly male (59\%). The median age was 63 years, and the older patients ( $\geq 70$ years of age) made up one-quarter of the study group. Almost the entire population had good performance statuses (97\% ECOG PS 0-1). More than one-half of the patients (55\%) suffered from colon cancer, while the rest suffered from rectal cancer. In most of the patients, distant metastases were diagnosed (86\%), and the liver was the most common site of involvement (42\% liver limited disease). With reference to the systemic treatment, only $30 \%$ were previously treated with adjuvant chemotherapy. The cytotoxic doublet (FOLFOX, CapeOX, or FOLFIRI) as the initial systemic therapy for advanced disease was administered in $73 \%$ of the patients.

Most of the patients had elevated serum CEA levels (78\%). Contrarily, the CA 19-9 sensitivity was lower and serum levels were increased only in every third person (34\%). The ALP and LDH levels were elevated in 30\% and $33 \%$, respectively. However, the listed laboratory data was not available for all of the patients, which limits the value of this analysis.

All of the patients had CBC data. A high white blood cell (WBC) count was associated with significantly worse PFS (HR $=1.76, p=0.0015)$ and OS $(H R=2.43$, $p<0.0001)$. This relationship was only found in the neutrophils (HR $=1.89, p=0.0004$ for PFS; $H R=2.51$, $p<0.0001$ for OS) and monocytes ( $\mathrm{HR}=1.38, p=0.016$ for PFS; HR = 1.82, $p<0.0001$ for OS). The lymphocyte count did not correlate with the survival $(H R=1.13$, $p=0.3609)$ nor the duration of disease control $(\mathrm{HR}=1.25, p=0.1115)$. As expected, those patients with anaemia had unfavourable prognoses $(H R=1.36$, $p=0.017$ for PFS; HR $=1.55, p=0.0005$ for OS). An elevated PLT also had a negative impact on the outcome, 
Table I. Results of univariate survival analysis

\begin{tabular}{lcccccc} 
Parameter & \multirow{2}{*}{ Compared value } & \multicolumn{2}{c}{ PFS } & OS \\
\cline { 4 - 7 } & & & HR $(95 \% \mathrm{Cl})$ & P-value & HR $(95 \% \mathrm{Cl})$ & $P$-value \\
\hline WBC & $\mathrm{N}$ & $>\mathrm{N}$ & $1.76(1.24-2.49)$ & 0.0015 & $2.43(1.73-3.40)$ & $<0.0001$ \\
\hline Neutrophils & $\mathrm{N}$ & $>\mathrm{N}$ & $1.89(1.33-2.68)$ & 0.0004 & $2.51(1.78-3.54)$ & $<0.0001$ \\
\hline Monocytes & $\mathrm{N}$ & $>\mathrm{N}$ & $1.38(1.06-1.79)$ & 0.016 & $1.82(1.40-2.36)$ & $<0.0001$ \\
\hline Lymphocytes & $\geq \mathrm{N}$ & $<\mathrm{N}$ & $1.25(0.95-1.63)$ & 0.1115 & $1.13(0.87-1.48)$ & 0.3609 \\
\hline Haemoglobin & $\mathrm{N}$ & $<\mathrm{N}$ & $1.36(1.06-1.74)$ & 0.017 & $1.55(1.21-1.98)$ & 0.0005 \\
\hline PLT & $\leq \mathrm{N}$ & $>\mathrm{N}$ & $1.39(1.01-1.92)$ & 0.043 & $1.85(1.34-2.55)$ & 0.0002 \\
\hline NLR & Low & High & $1.55(1.22-1.97)$ & 0.0004 & $1.88(1.48-2.38)$ & $<0.0001$ \\
\hline LMR & Low & High & $0.73(0.58-0.93)$ & 0.011 & $0.58(0.46-0.74)$ & $<0.0001$ \\
\hline PLR & Low & High & $1.25(0.98-1.58)$ & 0.07 & $1.39(1.10-1.76)$ & 0.0054
\end{tabular}

PFS - progression-free survival, OS - overall survival, HR - hazard ratio, Cl-confidence intervals, WBC - white blood cells, N-normal value, PLT-platelets, $N L R$ - neutrophil-to-lymphocyte ratio, LMR - lymphocyte-to-monocyte ratio, PLR - platelet-to-lymphocyte ratio.

especially on the OS $(H R=1.85, p=0.0002)$. Overall, our study confirmed the prognostic value of the parameters calculated on the basis of a CBC result. A high NLR and PLR were associated with shorter survival $(H R=1.88, p<0.0001$ for $N L R ; H R=1.39, p=0.0054$ for $P L R)$. For the $P L R$, we observed only a not significant trend toward a worse PFS (HR $=1.25, p=0.07$ for PLR, $\mathrm{HR}=1.55, p=0.0004$ for NLR). A high LMR was correlated with a better prognosis $(\mathrm{HR}=0.73, p=0.011$ for PFS; $\mathrm{HR}=0.58, p<0.0001$ for OS). The results of the univariate survival analysis are presented in Table I.

\section{Discussion}

These encouraging results forced us to deliberate over the mechanisms responsible for the relationships between the neutrophil, monocyte, lymphocyte, and platelet counts in the peripheral blood and carcinogenesis. Unfortunately, these processes are still poorly understood. There are a great deal of data indicating the critical role of the local immune response in carcinogenesis, but the systemic inflammatory response also seems to take part in this process [7]. A CBC, in addition to the concentrations of the $\mathrm{C}$-reactive protein (CRP) and some cytokines (interleukin (IL)-1ra, IL-6, IL-7, IL-8, IL-9, IL-12, interferon $\gamma$, interferon $\gamma$-induced protein 10 , monocyte chemotactic protein 1, macrophage inflammatory protein $1 \beta$ and platelet-derived growth factor $B B)$, as well as hypoalbuminaemia, indirectly reflects the activity of the immune system [8-10]. Moreover, CBC disorders in cancer patients may be involved in different aspects of carcinogenesis, including neoangiogenesis, CTCs, immune escape, infiltration, and metastases formation.
Neutrophilia is recognised as an indicator of the systemic inflammatory response, and this condition is associated with delayed neutrophil apoptosis. The circulating granulocyte-macrophage colony-stimulating factor is a significant inhibitor of neutrophil apoptosis in patients with systemic inflammatory response syndrome when IL-10 counters the process. These cytokines appear to modulate neutrophil apoptosis by altering reactive oxygen species generation in the neutrophils [11]. As well as the hypothesis explaining the mechanism of neutrophilia, there are studies concerned with its role in carcinogenesis. It is suspected that neutrophils assist in metastasis by suppressing peripheral leukocyte activation, which contributes to the circulating tumour cells' (CTCS) survival [12]. Furthermore, the interaction with the neutrophils enables the CTCS to enter the endothelium as the first step of metastasis formation [13].

Shibutani et al., in their study encompassing patients with stage II/III CRC, revealed that the peripheral monocyte count was positively associated with the density of the tumour-associated macrophages, and that a high value was correlated with an unfavourable prognosis [14]. There is a hypothesis suggesting that cancer cells produce chemokines (such as CCL2) that recruit monocytes from the peripheral blood to the tumour [15]. This, in turn, activates the passage of monocytes from the bone marrow to the peripheral blood. Thanks to the cytokines present in the cancer microenvironment (macrophage colony-stimulating factor, transforming growth factor- $\beta$ (TGF- $\beta$ ), IL- 6 , and IL-10), white cells differentiate into two phenotypes of macrophages: antitumour $M 1$ and procarcinogenic $M 2$ [16]. The latter 
prevail in the tumour microenvironment and produce angiogenic factors (vascular endothelial growth factor (VEGF) and tumour necrosis factor- $\alpha(T N F-\alpha)$ ) so that they facilitate neoangiogenesis and matrix metalloproteinase-dependent tumour invasion.

Lymphopaenia is often observed in those patients with advanced cancer, and it has a negative prognostic value for survival [17]; however, its mechanism is still poorly understood. There is one hypothesis that lymphopaenia may reflect pre-existing host immunosuppression [18]. Cancer cells may also contribute to lymphocyte destruction [19] or the disarrangement of their pools, and the latter process results from the overproduction of numerous cytokines and mediators and the impaired function of dendritic cells [20]. This hypothesis is supported by the observation that lymphopaenia coexists with inflammatory symptoms in patients with disseminated cancer. Bone marrow metastases and cachexia may also contribute to lymphopaenia [17].

Thrombocytosis can be seen in patients with different solid tumours, and it is often a negative prognostic factor. It has been suggested that there is a reciprocal interaction between cancer cells and platelets, which works as a positive feedback loop. Tumour cells directly and indirectly activate the platelets via thrombin generation. They also overexpress certain cytokines (IL-1, IL-3, IL-6, IL-11, leukaemia inhibitory factor, KitL, and oncostatin $M$ ) that play roles in tumour-stimulated thrombopoiesis [21]. Moreover, the activated platelets assist in tumour progression and may protect the CTCs from immune surveillance (via the production of platelet-derived growth factor and TGF- $\beta$ ) [22] and promote their extravasation and seeding, thanks to the adhesion molecules ( $\mathrm{P}$ - and L-selectins) [23]. The platelets can regulate neoangiogenesis by releasing the proangiogenic and antiangiogenic proteins contained in their $\alpha$ granules, which is essential in metastasis formation [24]. Moreover, the TGF- $\beta$ produced by the platelets probably contributes to the epithelial-to-mesenchymal transition in tumour metastasis [21, 22].

Our study focused on an evaluation of the bloodbased parameters as predictors of the response to systemic treatment or the long-term outcome. It is worth underscoring the fact that the complex markers: NLR, $L N R$, and PLR have strong prognostic value. We confirmed that a high NLR and a high PLR was a significant prognostic factor for poor OS, and our findings are in agreement with the results of several previous meta-analyses $[3,5,6,25,26]$. Contrary to the very heterogeneous population included in the above-mentioned meta-analyses (different tumour stages and various treatment methods), we concentrated on those patients with advanced CRC who qualified for the first line of chemotherapy. One important limitation was the lack of an optimal cut-off value. We divided the population using a median, which cannot be used in routine practice. However, various values were proposed in the other studies.

Contrary to the NLR and PLR, a high LMR value has a favourable impact on the outcome, and our results are in line with those previously published in the literature $[4,27]$. Additionally, considering the LMR, previous studies have been concerned mainly with resectable CRC [27]. However, we demonstrated that it is a significant prognostic marker for the OS and PFS in advanced CRC.

The strengths of our trial include its quite homogenous population, the evaluation of different bloodbased prognostic markers (at the same time), and the long follow-up period. However, our study also has several important limitations. It was a retrospective single-centre trial, so we cannot fully exclude selection bias. Moreover, we had no possibility of assessing the potential confounding factors, which might affect the neutrophil, lymphocyte, platelet, and monocyte counts, such as local or systemic infection and other chronic diseases. The patients were treated with chemotherapy only, and not with biologics, and at that time the bevacizumab and anti-epidermal growth factor receptor (anti-EGFR) antibodies were not reimbursed in the first-line treatment of CRC in our country. Considering the limitations of our study, well-designed prospective studies with an extended analysis of the abovementioned aspects of carcinogenesis are required to verify the results and apply them in clinical practice.

This study was not supported by the pharmaceutical industry.

\section{Conclusions}

The CBC parameters are readily available, reliable, and low-cost prognostic and predictive biomarkers, which can be easily incorporated into routine practice to predict the prognosis and to help determine the optimal treatment strategy for patients with metastatic CRC.

\section{Conflict of interest}

The authors declare no conflict of interest.

\section{References}

1. Wojciechowska U, Olasek P, Czauderna K, et al. Nowotwory złośliwe w Polsce w 2014 roku. Krajowy Rejestr Nowotworów, Warsaw 2016.

2. Van Cutsem E, Cervantes A, Adam R, et al. ESMO consensus guidelines for the management of patients with metastatic colorectal cancer. Ann Oncol 2016; 27: 1386-422.

3. Tang H, Li B, Zhang A, et al. Prognostic significance of neutrophil-to-lymphocyte ratio in colorectal liver metastasis: 
a systematic review and meta-analysis. PLoS One 2016; 11 e0159447.

4. Wu Q, Hu T, Zheng E, et al. Prognostic role of the lymphocyte-to-monocyte ratio in colorectal cancer: an up-to-date meta-analysis. Medicine (Baltimore) 2017; 96: e7051.

5. Gu X, Gao XS, Qin S, et al. Elevated platelet to lymphocyte ratio is associated with poor survival outcomes in patients with colorectal cancer. PLoS One 2016; 11: e0163523.

6. Chen N, Li W, Huang K, et al. Increased platelet-lymphocyte ratio closely relates to inferior clinical features and worse long-term survival in both resected and metastatic colorectal cancer: an updated systematic review and meta-analysis of 24 studies. Oncotarget 2017; 8: 32356-69.

7. Fridman WH, Pagès F, Sautès-Fridman C, Galon J. The immune contexture in human tumours: impact on clinical outcome. Nat Rev Cancer 2012; 12: 298-306.

8. Mantovani A, Allavena P, Sica A, Balkwill F. Cancer-related inflammation. Nature 2008; 454: 436-44.

9. Candido J, Hagemann T. Cancer-related inflammation. J Clin Immunol 2013; 33 Suppl 1: S79-84.

10. Lin WW, Karin M. A cytokine-mediated link between innate immunity, inflammation, and cancer. J Clin Invest 2007; 117: 1175-83.

11. Fanning NF, Kell MR, Shorten GD, et al. Circulating granulocyte macrophage colony-stimulating factor in plasma of patients with the systemic inflammatory response syndrome delays neutrophil apoptosis through inhibition of spontaneous reactive oxygen species generation. Shock 1999; 11: 167-74.

12. Zhang J, Qiao X, Shi H, et al. Circulating tumor-associated neutrophils (CTAN) contribute to circulating tumor cell survival by suppressing peripheral leukocyte activation. Tumour Biol 2016; 37: 5397-404.

13. Tao L, Zhang L, Peng Y, et al. Neutrophils assist the metastasis of circulating tumor cells in pancreatic ductal adenocarcinoma: a new hypothesis and a new predictor for distant metastasis. Medicine (Baltimore) 2016; 95: e4932.

14. Shibutani M, Maeda K, Nagahara H, et al. The peripheral monocyte count is associated with the density of tumor-associated macrophages in the tumor microenvironment of colorectal cancer: a retrospective study. BMC Cancer 2017; 17: 404

15. Szebeni GJ, Vizler C, Kitajka K, Puskas LG. Inflammation and cancer: extra- and intracellular determinants of tumor-associated macrophages as tumor promoters. Mediators Inflamm 2017; 2017: 9294018

16. Sica A, Larghi P, Mancino A, et al. Macrophage polarization in tumour progression. Semin Cancer Biol 2008; 18: 349-55.

17. Ray-Coquard I, Cropet C, Van Glabbeke M, et al. European Organization for Research and Treatment of Cancer Soft Tissue and Bone Sarcoma Group. Lymphopenia as a prognostic factor for overall survival in advanced carcinomas, sarcomas, and lymphomas. Cancer Res 2009; 69: 5383-91.

18. Caras I, Grigorescu A, Stavaru C, et al. Evidence for immune defects in breast and lung cancer patients. Cancer Immuno Immunother 2004; 53: 1146-52.

19. Strand S, Hofmann WJ, Hug H, et al. Lymphocyte apoptosis induced by CD95 (APO-1/Fas) ligand-expressing tumo cells - a mechanism of immune evasion? Nat Med 1996; 2: $1361-6$.
20. Martin F, Apetoh L, Ghiringhelli F. Controversies on the role of Th17 in cancer: a TGF-beta-dependent immunosuppressive activity? Trends Mol Med 2012; 18: 742-9.

21. Lin RJ, Afshar-Kharghan V, Schafer Al. Paraneoplastic thrombocytosis: the secrets of tumor self-promotion. Blood 2014; 124: 184-7.

22. Tesfamariam B. Involvement of platelets in tumor cell metastasis. Pharmacol Ther 2016; 157: 112-9.

23. Kappelmayer J, Nagy B Jr. The interaction of selectins and PSGL-1 as a key component in thrombus formation and cancer progression. Biomed Res Int 2017; 2017: 6138145.

24. Battinelli EM, Markens BA, Kulenthirarajan RA, et al. Anticoagulation inhibits tumor cell-mediated release of platelet angiogenic proteins and diminishes platelet angiogenic response. Blood 2014; 123: 101-12

25. Malietzis G, Giacometti M, Kennedy RH, et al. The emerging role of neutrophil to lymphocyte ratio in determining colorectal cancer treatment outcomes: a systematic review and metaanalysis. Ann Surg Oncol 2014; 21: 3938-46.

26. Guo YH, Sun HF, Zhang YB, et al. The clinical use of the platelet/lymphocyte ratio and lymphocyte/monocyte ratio as prognostic predictors in colorectal cancer: a meta-analysis. Oncotarget 2017; 8: 20011-24.

27. Stotz M, Pichler M, Absenger G, et al. The preoperative lymphocyte to monocyte ratio predicts clinical outcome in patients with stage III colon cancer. Br J Cancer 2014; 110: 435-40.

Received: 24.10.2017

Accepted: 24.04 .2018 